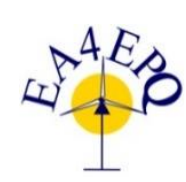

International Conference on Renewable Energies and Power Quality (ICREPQ'18)

Salamanca (Spain), 21 th $23^{\text {th }}$ March, 2018

Renewable Energy and OPour Qualily. Gournal (RE\&PQJ)

ISSN 2172-038 X, No.16 April 2018

\title{
Power Calculation Algorithm for Single-Phase Droop-Operated Inverters Considering Nonlinear Loads and unsing n-Order SOGI Filtering
}

\author{
J. El Mariachet†, J. Matas $\uparrow$, J. de la Hoz†, Y. Al-Turki*, H. Abdalgader* \\ $†$ Department of Electric Engineering, Universidad Politécnica de Cataluña (UPC), Barcelona, Spain. \\ *Renewable Energy Research group, King Abdulaziz University (KAU), Jeddah, Saudi Arabia.
}

\begin{abstract}
The average active and reactive powers, $P$ and $Q$, are crucial parameters that have to be calculated when sharing common loads between parallelized droop-operated single-phase inverters. However, the droop method algorithm should employ low-pass filters (LPF) with very low cut-off frequency to minimize the distortion impact in the provide droop amplitude and frequency references. This situation forces the droop control to operate at a very low dynamic velocity, degrading the stability of the parallelized system. For this reason, different solutions had been proposed in literature to increase the droop velocity, but the issues derived from the sharing of nonlinear loads had not been properly considered. This work proposes a novel method to calculate $P$ and $Q$ based on the fundamental components of the inverter's output voltage and current and using the measured phase angle between the output voltage and current. The method is used under normal and highly distorting conditions due to the sharing non-linear loads. The fundamental components are obtained by means of the highly filtering capability provided by $n$ order cascaded second order generalized integrators (nSOGI). The proposed method leads to faster and more accurate $P$ and $Q$ calculations that enhances the droop-method dynamic performance. Simulations are provided to validate the proposal.
\end{abstract}

Keywords-component; Active and reactive power calculation, singlephase inverters, nonlinear loads, inverter parallelization, droop method, harmonic distortion, power quality.

\section{INTRODUCTION}

The calculation of the averaged active, $P$, and reactive, $Q$, powers is an important aspect in the droop based local control algorithm used to parallel single-phase inverters without communication between units, since it has a critical influence on the transient response speed of the inverter and in the parallel system stability [1]-[3]. This calculation had been usually performed by the multiplications of the inverter delivered output current, $i_{O}(t)$, with the inverter output voltage, $v_{O}(t)$, and with the voltages $\pi / 2$ phase shifted version, $v_{o \perp}(t)$, for obtaining the active and reactive instantaneous powers, $p_{i}(t)$ and $q_{i}(t)$, respectively. In the droop-method usually a LPF should be applied to achieve the averaged values of $p_{i}(t)$ and $q_{i}(t)$ and for removing the double frequency component resulting for the multiplication of these sinusoidal signals when sharing linear loads [4]-[12]. In this operation, $v_{o \perp}(t)$ can be obtained by different approaches such as a transport delay (TD) in [13] and [14], an extended three-phase $d q \mathrm{SRF}$ approach applied to single-phase systems in [15]-[16], and a method using the quadrature output of a SOGI filter in [17]. In [18] another method based on the calculation of powers and later cancellation of the double frequency component extracted by means of a SOGI, similarly to [6], was proposed. This method used a LPF and showed to reduce the time needed for calculating the powers in one order of magnitude, but using only linear loads did not consider nonlinear loads. In [19] a proposal based on a discrete Fourier transform (DFT) was made for deriving the averaged powers. However, this approach introduced a severe delay that makes it unsuitable for load having abrupt perturbations. In [20] a LMS algorithm was introduced for obtaining $P$ and $Q$, but used approximations that only consider steady state conditions and consider only linear loads. These proposals have in common the objective of achieving a fast and accurate calculation of the averaged powers for generating the droop references in voltage and frequency. However, the validity of these approaches is only partial when sharing nonlinear loads or under harmonic voltage pollution. This paper proposes a modification in the power evaluation schemes shown in [6] and [18], using a n-order SOGI (nSOGI) approach for obtaining the fundamental components of the inverter output voltage and current when sharing a nonlinear load and under a $3^{\text {rd }}$ harmonic and 5\% amplitude voltage pollution. The method uses the amplitude of the voltage and current fundamental components and the measured phase angle between the voltage and current for the calculation of $P$ and $Q$. The filtering capability of the nSOGI filter is determined by its damping factor parameter, $\xi$, which for minimizing the ripple in the obtained powers to a predefined desired value. The nSOGI proposal leads to faster $P$ and $Q$ calculations, since overcomes the speed limitations that other approaches have due to the LPF used in their structure. Comparisons with the classical droop method and with the methods proposed in [6] and [18] are made under the assumption of causing the same amplitude ripple at the final derived averaged powers when using a $\mathrm{R}-\mathrm{C}$ rectified type nonlinear load.

This paper is organized as follows, in Section II the power calculation block used in a classical droop method for a singlephase inverter is described. In Section III an advanced method for calculating $P-Q$ based on [18] is shown exposing the problems when using nonlinear loads and harmonic voltage distortion. Section IV proposes the novel power calculation algorithm using the fundamental components using the nSOGI 
approach and showing simulation results for validating the proposal. Section V presents the conclusions of this work.

\section{POWER CALCULATION IN SINGLE-PHASE DROOP- OPERATED INVERTERS}

Fig. 1 illustrates a basic scheme of a single-phase inverter that is operated with the droop method. In this figure can be seen that the control scheme is composed by a $P-Q$ power calculation block, a voltage reference extraction block named after "droop method", and the inverter's control inner loops plus pulse width modulation (CTRL+PWM) block. The $P-Q$ block uses the inverter's output voltage and current to calculate the averaged powers, $P_{a v}$ and $Q_{a v}$, to generate the inverter's voltage reference, $v_{r e f}$, to command the inverter's switches through the inner loops plus PWM control block.

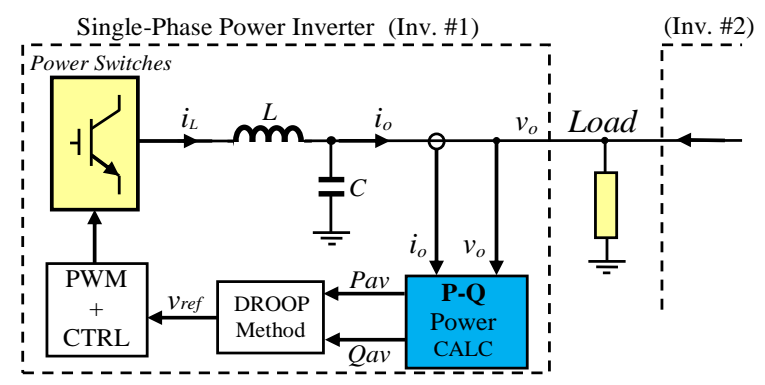

Fig. 1. Generic Droop-based control scheme of a single-phase inverter

Fig. 2 shows the traditional power calculation method that multiplies the output voltage and current for obtaining the instantaneous active, $p i$, and reactive, $q i$, powers. The method uses LPFs at the end for obtaining the averaged powers [17].

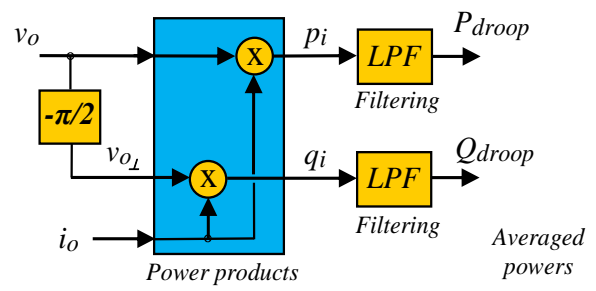

Fig. 2 Block diagram of conventional averaged P-Q power calculation method.

The droop control method determines the proper operating frequency and amplitude voltage for the inverter through the following equations, when line impedance and output inverter's impedance are considered to be mainly inductive:

$$
\begin{aligned}
& \omega^{*}=\omega_{n}-m \cdot \Delta P \\
& V^{*}=V_{n}-n \cdot \Delta Q
\end{aligned}
$$

where $m$ and $n$ are the droop coefficients, $\omega_{n}$ and $\omega^{*}$ are the nominal output and rated frequencies of the inverter, $V_{n}$ and $V^{*}$ are the nominal output and rated amplitudes of the inverter, respectively. The droop method uses (1)-(2) for driving the following sinusoidal voltage reference

$$
v_{\text {ref }}=V^{*} \sin \left(\omega^{*} t\right)
$$

Assuming that the inverter has no distortion [15], the output voltage and current can be described by

$$
\begin{aligned}
& v_{o}(t)=V \cdot \sin \left(\omega_{0} t\right) \\
& i_{o}(t)=I \cdot \sin \left(\omega_{0} t-\varphi_{o}\right)
\end{aligned}
$$

where $V$ and $I$ are the voltage and current amplitudes, $\omega_{o}$ is the fundamental frequency and $\varphi_{o}$ is the phase angle between $v_{o}$ and $i_{o}$. The quadrature voltage is defined as

$$
v_{o \perp}(t)=V \cdot \sin \left(\omega_{o} t-\frac{\pi}{2}\right)
$$

So, the instantaneous active and reactive powers could be formulated as

$$
\begin{gathered}
p_{i}=v_{o}(t) \cdot i_{o}(t)=\frac{V I}{2} \cdot\left[\cos \varphi_{o}-\cos \left(2 \omega_{0} t-\varphi_{o}\right)\right]= \\
=P+\tilde{p}
\end{gathered}
$$

And, in a similar way,

$$
\begin{gathered}
q_{i}=v_{o \perp}(t) \cdot i_{o}(t)=\frac{V I}{2} \cdot\left[\sin \varphi_{o}-\sin \left(2 \omega_{0} t-\varphi_{o}\right)\right]= \\
=Q+\tilde{q}
\end{gathered}
$$

where $P$ and $Q$ are the average active and reactive powers and $\tilde{p}$ and $\tilde{q}$ are the oscillating components at twice of the fundamental operating frequency provided by the droop method.

The LPFs used to filter $p_{i}$ and $q_{i}$, see Fig. 2, should have a low cut-off frequency value, $f c$, in order to reject the double frequency components, $\tilde{p}$ and $\tilde{q}$, and achieve the averaged values. The cut-off frequency should be of one or two order of magnitude lower than the inverter's operating frequency [21], [22]. The $f c$ value determines finally the speed of the droop method, which is too slow, typically less than $1 \mathrm{~Hz}$. In the case of sharing nonlinear loads, $f c$ should be reduced even more to handle the induced high distortion. The distortions induce by nonlinear loads can induce distortion in the inverter's output voltages. So, the $P-Q$ powers are strongly affected by nonlinear currents and also by the induced distortions in the output voltage, [23]. Therefore, it is crucial to keep the ripple distortion low in order not to disturb too much the droop-method's frequency and amplitude references, $\omega^{*}$ and $V^{*}$, which can cause a bad operation for the paralleled system.

\section{ADVANCED P-Q POWER CALCULATION METHOD}

A SOGI is a special linear filter that delivers two output signals, $v_{d}$ and $v_{q}$, which are in-phase and $\pi / 2$ delayed with respect to the input signal, $v_{i n}$, which have the following BPF and LPF transfer functions relationship regarding the input, respectively[25]

$$
\begin{gathered}
H_{d}(s)=\frac{v_{d}(s)}{v_{i n}(s)}=\frac{2 \xi_{i} \omega_{i}^{2} \cdot s}{s^{2}+2 \xi_{i} \omega_{i} \cdot s+\omega_{i}^{2}} \\
H_{q}(s)=\frac{v_{q}(s)}{v_{i n}(s)}=\frac{2 \xi_{i} \omega_{i}^{2}}{s^{2}+2 \xi_{i} \omega_{i} \cdot s+\omega_{i}^{2}}
\end{gathered}
$$

where $\xi_{i}$ is the filter damping factor and $\omega_{i}$ its tuning center frequency. These two parameters determine the settling time of the transient response of this filter

$$
t_{s} \approx \frac{4}{\xi_{i} \omega_{i}}
$$

The magnitude of (9) for a given harmonic $h$ of the fundamental frequency $\omega_{o}$

$$
\left|H_{d}\left(j h \omega_{o}\right)\right|=\frac{2 \xi \cdot h}{\sqrt{\left(1-h^{2}\right)^{2}+(2 \xi \cdot h)^{2}}}
$$

where $0<\xi<1$ and $h$ is an integer number. Taking into account the typical $h$ values 3, 5, 7, and so on, then (12) can be further simplified to

$$
\left|H_{d}\left(j h \omega_{o}\right)\right| \approx \frac{2 \xi}{h}
$$

From (11)-(13) can be seen that the SOGI has a trade-off relationship between harmonic attenuation and settling time, i.e. for small values of $\xi<<1$ has a strong harmonic attenuation, but at an expense of a very long settling time. Taking profit of these $\mathrm{BPF}$ characteristics for only linear loads and considering the 
drawbacks of the described conventional $P-Q$ calculation, an attempt to cancel the double frequency pulsations in (7)-(8) was proposed in [18]. This method was intended to accelerate the calculation of the active and reactive powers, as shown in the Fig. 3 block scheme:

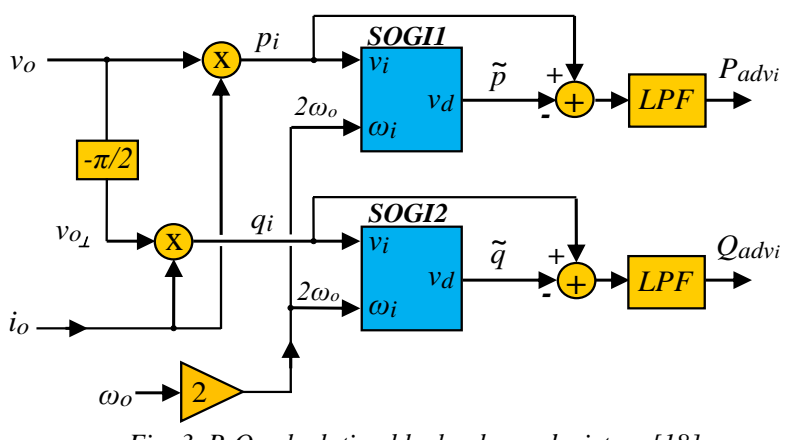

Fig. 3. P-Q calculation block scheme depict on [18].

In Fig. 3, SOGI1 and SOGI2 are used for extracting the pulsating double frequency components, $\tilde{p}$ and $\tilde{q}$, respectively. These SOGI are tuned both at $\omega_{i}=2 \omega_{0}$ and $\xi_{1}=\xi_{2}=1$. The purpose of the LPF of the final stage is to provide the averaged powers $P_{a d v i}$ and $Q_{a d v i}$. Fig. 3 do not show the method for generating the $\pi / 2$ delay since is not mentioned in [18]. Therefore another special linear filter, SOGI0, tuned at $\omega_{i}=\omega_{0}$ and $\xi=0.707$, is used for generating this phase-shift as shown in Fig. 4, for avoiding the undesired delay issues reported in [13], [14] and [19]. This method will be named as Advanced Method, although is not the focus of this work. The obtained averaged $P-Q$ are then named as $P_{a d v}$ and $Q_{a d v}$.

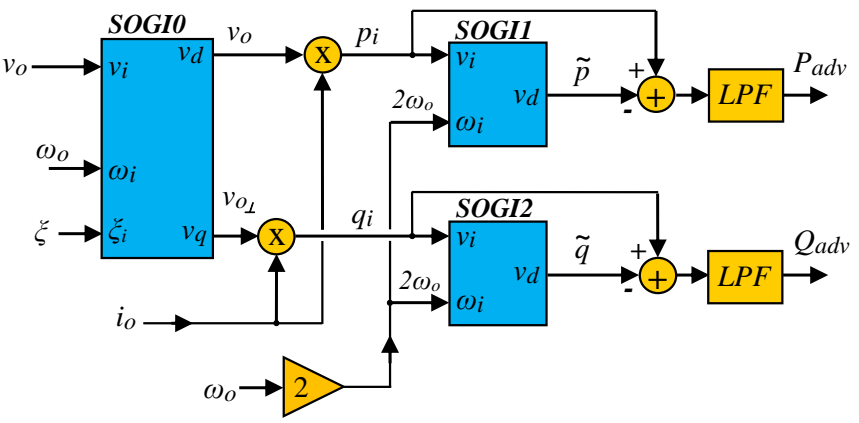

Fig. 4. Advanced P-Q calculation block scheme of Fig. 3 using an additional SOGI for generating the $\pi / 2$ delay.

Fig. 5 shows the simulations results after using the P-Q scheme of Fig. 4 when sharing a linear load that produces a current perturbation from $4 \mathrm{~A}$ to $8 \mathrm{~A}$ peak at time $1 \mathrm{~s}$, without distortion pollution in voltage. The dynamics of the active calculated power, $P_{a d v}$, is compared with those of the obtained active powers by the conventional droop method in Fig. 2, named as $P_{\text {droop }}$ and $Q_{\text {droop. }}$. The cut-off frequency of the LPF in the conventional scheme is set to $f_{c}=1 \mathrm{~Hz}$ and for the advanced method in fig. 4 the final LPF has $f c=10 \mathrm{~Hz}$.

As expected, the advanced method removes faster and better the double frequency component in the power in front of linear loads. Note that only Pdroop has a small ripple due to the double frequency component. These results are compatible with those reported in [18], only referred to active power against abrupt linear loads changes. However, these good results change when a nonlinear load is used as can be seen in Fig. 6. A nonlinear load $\mathrm{RC}$ rectifier type that draws a highly distorted current with $4 \mathrm{~A}$ peak is used. At time $1 \mathrm{~s}$ a change in the nonlinear load is induced that pushes the peak to $8 \mathrm{~A}$. The simulation parameters are shown in Table I.
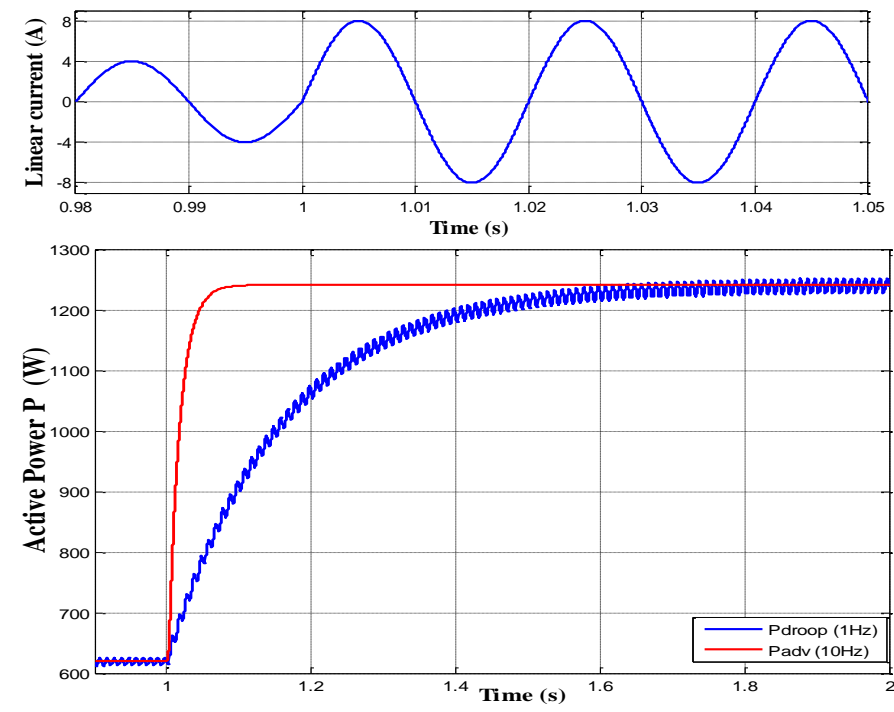

Fig. 5. $P_{\text {droop }}$ and $P_{\text {adv }}$ transient response for a linear load current perturbation from $4 A$ to $8 A$ at 1s: a) Detail of the perturbation; b) $P_{\text {droop }}$ and $P_{\text {adv }}$ calculated powers.

TABLE I. SIMULATION PARAMETERS FOR FIG. 6.

\begin{tabular}{c|c}
\hline$V_{n}$ & $311 \mathrm{~V}$ \\
$\omega_{n}$ & $2 \pi 50(\mathrm{rad} / \mathrm{s})$ \\
$R$ at $\mathrm{t}<1 \mathrm{~s} ; R$ at $\mathrm{t}>1 \mathrm{~s}$ & $1100 \Omega ; 372 \Omega$ \\
$\mathrm{C}$ & $470 \mu \mathrm{F}$ \\
$\xi_{0}$ & 0.7 \\
$\xi_{1}, \xi_{2}$ & 1 \\
$f c_{d r o o p} ; f_{a d v}$ & $1 \mathrm{~Hz} ; 10 \mathrm{~Hz}$ \\
\hline
\end{tabular}
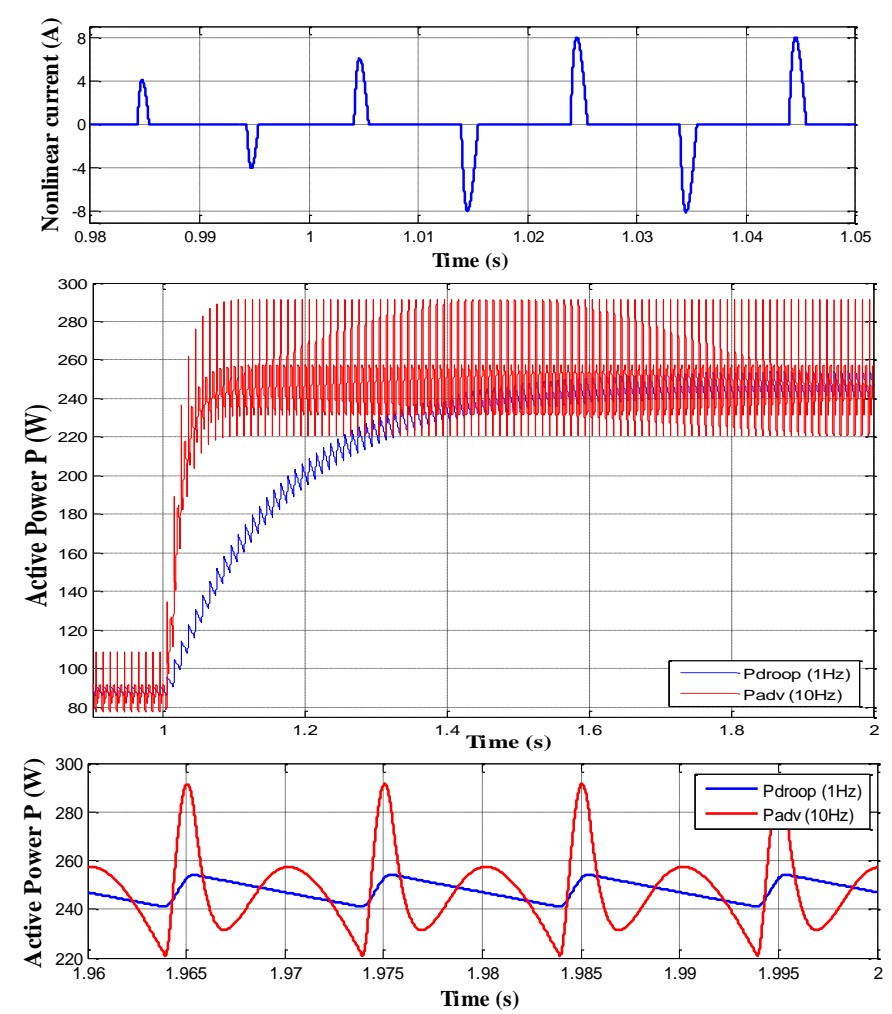

Fig. 6. $P_{\text {droop }}$ and $P_{\text {adv }}$ transient response for a nonlinear rectifier-type load perturbation in current from $4 A$ peak to $8 A$ peak at $1 \mathrm{~s}:$ a) Detail of the distorted load current perturbation from $4 A$ peak to $8 A$ peak; $b) P_{\text {droop }}$ and $P_{a d v}$ calculated powers. c) Detail of calculated powers showing their ripple. 
The dynamics of the proposed method in [18] were never considered using a nonlinear load, similarly to other proposals mentioned in Section I. Thus, in the presence of nonlinear loads the method has excessive steady state ripple due to the distortion corrupting the calculated powers, oppositely to the stated in [18]. For this reason, $f_{c}$ of the LPF in the advanced scheme of Fig. 4 was reduced to $f c=2.2 \mathrm{~Hz}$, for diminishing the ripple of $P_{a d v}$ until the same level than the conventional droop method is achieved. Fig. 7a and Fig. 7b show this situation and also how the advanced method is still faster calculating $P$ and $Q$ than the conventional droop controller. Nevertheless, although the advantages performed by the advanced method, under the same dynamical and distortion-attenuation conditions, it demonstrates to be less effective than initially argued.
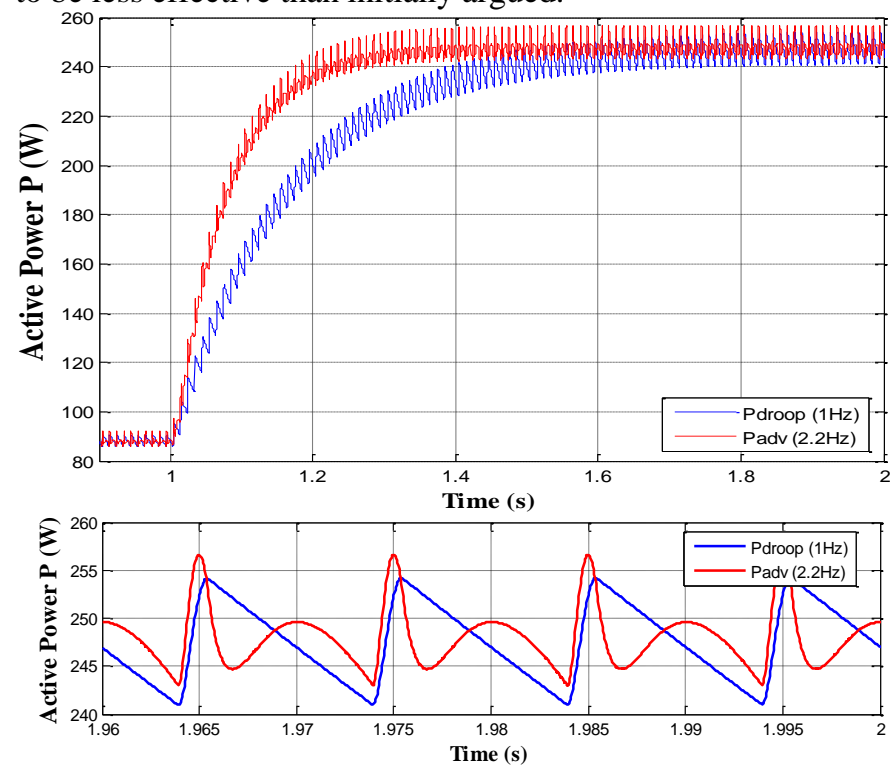

Fig. 7. $P_{\text {droop }}$ and $P_{\text {adv }}$ transient response for a nonlinear rectifier-type load perturbation from 4A peak to 8 A peak at 1s: a) Pdroop and Padv calculated powers, for $P_{a d v}$ with a $L P F$ with $f c=2.2 \mathrm{~Hz}$; b) Detail of the calculated powers showing their ripple;

Note also in Fig. 7a that there is a positive offset only for the calculated $P_{\text {droop }}$ at steady state, since the mean value of $P_{a d v}$ is slightly higher. It is so because the conventional method does not calculate well the averaged power in steady state conditions when a nonlinear load is used. In the other hand, a harmonic pollution in the voltage can alter the calculus of $P-Q$. Fig. 8 depicts the distortion induced for a grid voltage having only a $3^{\text {th }}$ harmonic distortion of 5\% amplitude and Fig 9 illustrates the result of having this pollution in voltage plus the nonlinear load of Fig. 7. Note that in Fig. 8 the $3^{\text {rd }}$ harmonic distorts the conventional droop method calculated power which is more degraded in Fig. 9, where it is also shown an error in the droop averaged power. This error, caused by the use of a transport delay to generate the $90^{\circ}$ voltage delay for the droop power calculation, disappears if a SOGI filtering is adopted instead.

\section{PROPOSED $P$ - $Q$ nSOGI POWER CALCULATION METHOD} In order to avoid the previous problems a novel algorithm is proposed and shown in Fig. 10. Considering the advanced method using a nonlinear load, the output time domain current of the inverter in steady state can be expressed [23] as:

$i_{o}(t)=I_{D C}+I_{0} \cdot \sin \left(\omega_{o} t+\varphi_{o}\right)+\sum_{h=2}^{N} I_{h} \cdot \sin \left(h \cdot \omega_{o} t+\varphi_{h}\right)$ where the sub index $\boldsymbol{h}$ represents the harmonic number, $\boldsymbol{N}$ the maximum set of harmonics, $I_{D C}$ the DC component, $I_{o}$ and $I_{h}$ are the amplitudes of the fundamental and harmonic components, respectively. The fundamental frequency is $\omega_{o}$ and $\left(h \cdot \omega_{o}\right)$ represents its harmonic multiples. Finally, $\varphi_{o}$ and $\varphi_{h}$ are the phase-shift for the fundamental and harmonic components, respectively. Also, the $3^{\text {rd }}$ harmonic voltage pollution can be expressed as:

$$
v_{o}(t)=V_{0} \cdot \sin \left(\omega_{o} t\right)+0.05 \cdot V_{0} \cdot \sin \left(3 \cdot \omega_{o} t+\theta_{3}\right)
$$

where $V_{0}$ is the voltage fundamental amplitude, $\omega_{o}$ the fundamental frequency and $\theta_{3}$ the $3^{\text {rd }}$ harmonic phase-shift . Thus, accordingly to the scheme proposed in Fig.4, the instantaneous powers should be redefined as:

$$
\begin{aligned}
p_{i}^{\prime}=P_{a v} & +\tilde{p}+v_{o}(\mathrm{t}) \cdot \sum_{h=2}^{N} I_{h} \cdot \sin \left(h \cdot \omega_{o} t+\varphi_{h}\right) \\
& +0.05 V_{0} \cdot \sin \left(3 \cdot \omega_{o} t+\theta_{3}\right) \cdot \sum_{h=1}^{N} I_{h} \sin \left(h \cdot \omega_{o} t+\varphi_{h}\right) \\
q_{i}^{\prime}=Q_{a v} & +\tilde{q}+v_{o \perp}(\mathrm{t}) \cdot \sum_{h=2}^{N} I_{h} \cdot \sin \left(h \cdot \omega_{o} t+\varphi_{h}\right) \\
& +0.05 V_{0} \cdot \sin \left(3 \cdot \omega_{o} t+\theta_{3}-\frac{\pi}{2}\right) \cdot \sum_{h=1}^{N} I_{h} \sin \left(h \cdot \omega_{o} t+\varphi_{h}\right)
\end{aligned}
$$

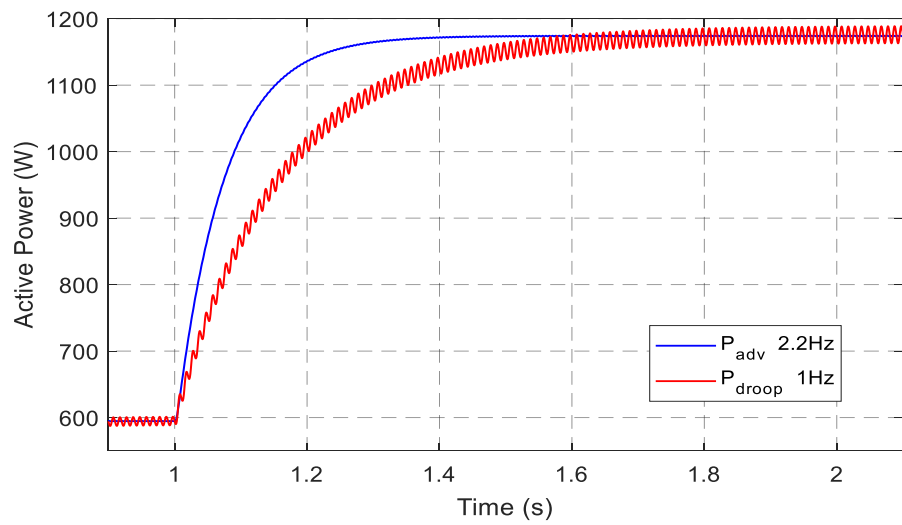

Fig. 8. $P_{\text {droop }}$ and $P_{\text {adv }}$ transient response for a linear load perturbation from $4 A$ peak to $8 A$ peak at $1 \mathrm{~s}$ and considering a $3^{\text {rd }}$ harmonic of $5 \%$ amplitude distortion in the supplied voltage.

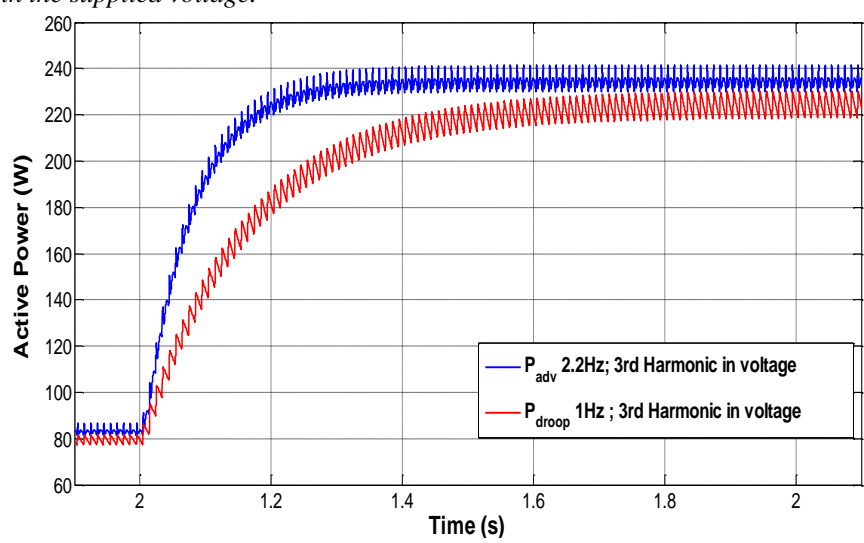

Fig. 9. Pdroop and Padv transient response for a nonlinear rectifier-type load perturbation from $4 A$ peak to $8 A$ peak at $1 s$ and considering a $3^{\text {rd }}$ harmonic of $5 \%$ amplitude distortion in the supplied voltage.

Therefore, the expressions for $p_{i}^{\prime}$ and $q_{i}^{\prime}$ become much more complex to be analyzed than $p_{i}$ and $q_{i}$. Here, (16) and (17) contain the averaged powers components, the pulsating double frequency components, also represented in (7) and (8), plus the product between the voltages and the harmonic components of the load current. In the case of (17) a $\frac{\pi}{2}$ delay is also introduced in the $3^{\text {rd }}$ harmonic. For sake of simplicity any harmonic phaseshift in voltage has been neglected. Thus, the subtraction of only 
the double frequency component driven in the advanced method is not enough for the proper calculation of $P_{a v}$ and $Q_{a v}$, since it becomes necessary the filtering of the measured current and voltage, $i_{o}$ and $v_{o}$, in order to reject their harmonic components.
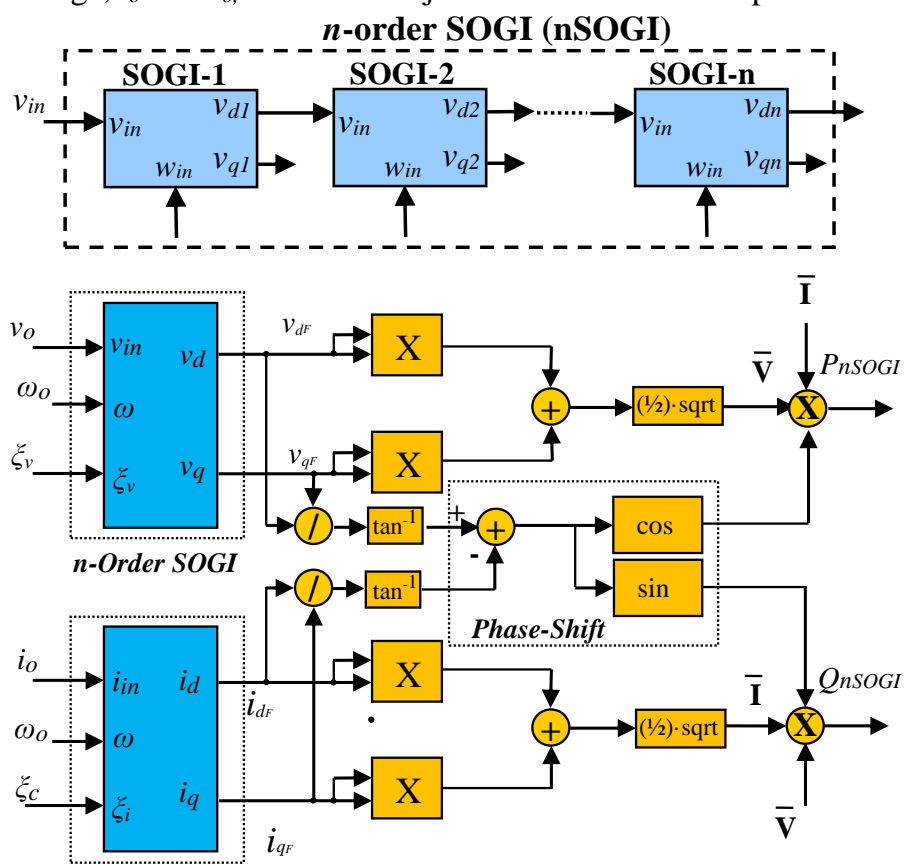

Fig.10. Scheme depicting the cascaded n-Order SOGI up) Scheme of a cascaded n-order SOGI structure; down) Proposed P-Q calculation method for dealing with nonlinear loads and using a nSOGI approach.

Fig.10 shows the nSOGI approach for the obtention of the voltage and current fundamental components. The first stage is achieved through $n$ general integrators cascaded, following the proposed one in [25]. Its transfer function is defined as:

$$
H_{d n}(s)=\frac{v_{d n}(s)}{v_{i n}(s)}=\left(\frac{2 \xi \omega_{o} \cdot s}{s^{2}+2 \xi \omega_{o} \cdot s+\omega_{o}^{2}}\right)^{n}
$$

From which the relationship between damping factor and attenuation to specified harmonic can be expressed as

$$
\xi \approx \frac{h}{2} \cdot\left|H_{d n}\left(j h \omega_{o}\right)\right|^{1 / n}
$$

where $\xi$ is the damping factor, $\left|H_{d n}\left(j h \omega_{0}\right)\right|$ the attenuation at the harmonic $h$ and $n$ is the filter order. Fig. 11 plots the filtering capability of the nSOGI as the filter order increases. The amplitude of the fundamental components are obtained as

$$
\begin{aligned}
& \bar{V}=\sqrt{\left|v_{o F}\right|^{2}+\left|v_{o F \perp}\right|^{2}} \\
& \bar{I}=\sqrt{\left|i_{\mathrm{OF}}\right|^{2}+\left|i_{\mathrm{OF} \perp}\right|^{2}}
\end{aligned}
$$

where $v_{0 F}$ and $i_{0 F}$ are the voltage and current fundamental components and $v_{0 F \perp}$ and $i_{0 F \perp}$ their respective $\pi / 2$ phaseshifted versions, respectively. By considering $\varphi$ as the phaseshift between fundamental voltage and current due to the presence of reactive, as defined in [23]-[24], the averaged active and reactive powers can be calculated as

$$
\begin{aligned}
\varphi & =\tan ^{-1}\left(v_{0 F \perp} / v_{0 F}\right)-\tan ^{-1}\left(i_{0 F} / i_{0 F \perp}\right) \\
P & =\frac{1}{2} \bar{V} \cdot \bar{I} \cdot \cos (\varphi) \\
Q & =\frac{1}{2} \bar{V} \cdot \bar{I} \cdot \sin (\varphi)
\end{aligned}
$$

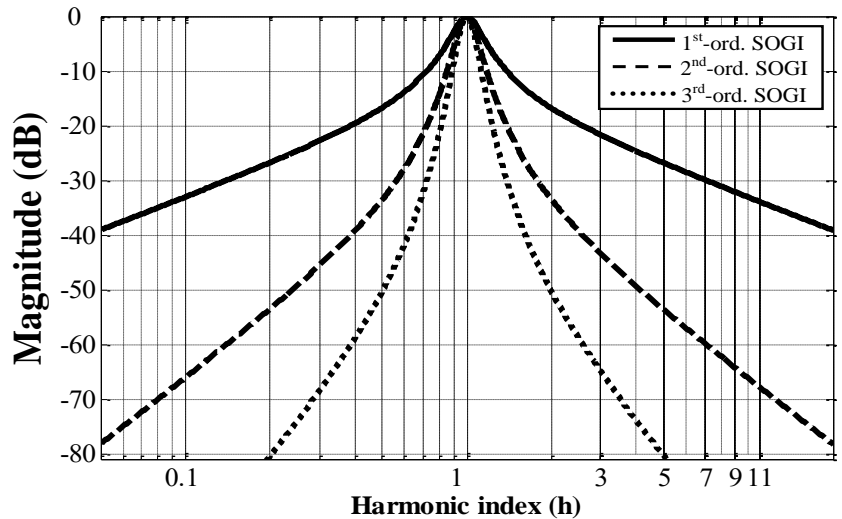

Fig. 11. Bode magnitude plot of a $1^{\text {st }}, 2^{\text {nd }}$, and $3^{\text {rd }}-$ order SOGI for $\xi=0.11$.

TABLE II. SimUlation PARAMETERS FIG.12.

\begin{tabular}{c||c}
\hline \hline \multicolumn{1}{|c||}{$\begin{array}{c}\text { Vo } \\
3^{\text {rd }} \text { harmonic voltage } \\
\text { amplitude }\end{array}$} & $311 \mathrm{~V}$ \\
$\omega_{0}$ & $5 \%$ \\
$R$ at $\mathrm{t}<1 \mathrm{~s} ; R$ at $\mathrm{t}>1 \mathrm{~s}$ & $2 \pi 50(\mathrm{rad} / \mathrm{s})$ \\
$C$ & $4700 \Omega ; 372 \Omega$ \\
$\xi_{v}$ & 0.7 \\
$\xi_{i}$ & 0.25 \\
$f c_{\text {droop } ;} f c_{a d v}$ & $1 \mathrm{~Hz} ; 2.2 \mathrm{~Hz}$ \\
\hline
\end{tabular}

TABLE III. SiMULATION RESULTS FIG. 12

\begin{tabular}{c|c}
\hline \hline \multicolumn{2}{c}{ Measured rise time in transient $(\mathrm{ms})$} \\
\hline \hline$P_{D R O O P}$ & 270.624 \\
$P_{A D V}$ & 127.321 \\
$P_{n S O G I}$ & 42.047 \\
\hline \hline \multicolumn{2}{c|}{ Improvement in rise time (reduction) } \\
\hline \hline$P_{n S O G I} V S P_{A D V}$ & $-66.975 \%$ \\
$P_{N S O G I} V S P_{D R O O P}$ & $-84.45 \%$ \\
\hline
\end{tabular}
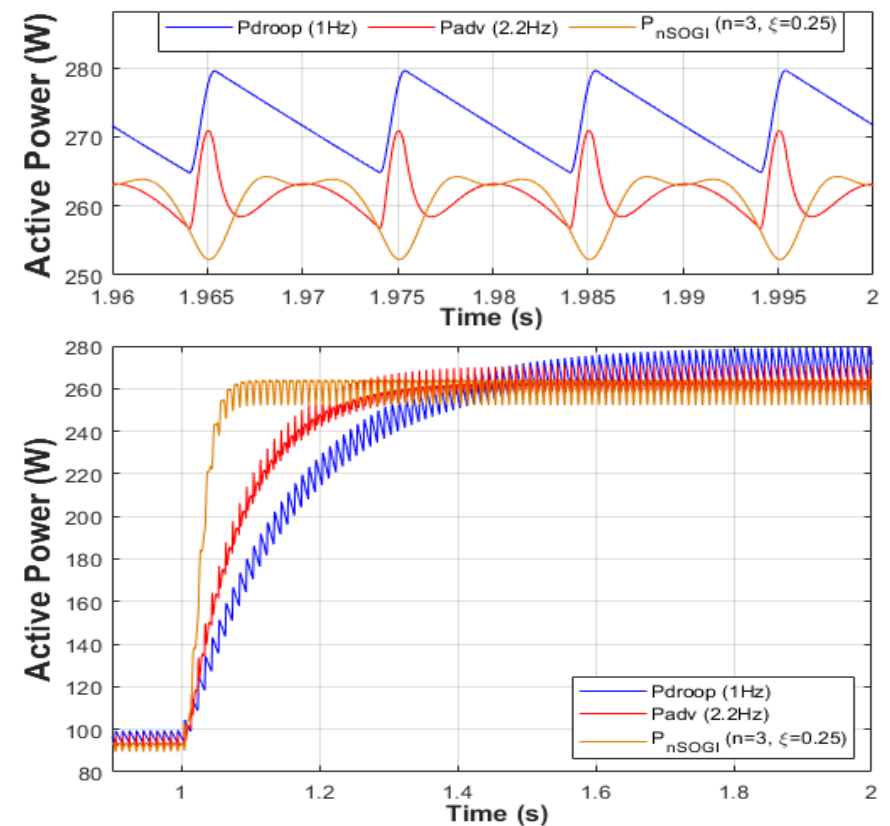

Fig. 12. Transient responses for a nonlinear rectifier-type load perturbation from $4 A$ peak to $8 A$ peak at $1 s$, with a $5 \%$ in amplitude $3^{\text {rd }}$ harmonic in voltage: up) Calculated powers at steady-state. down) Pdroop, Padv and $P_{n S O G I}$ active powers.

The resulting values will be referred as $P_{n S O G I}$ for the active power, and $Q_{n S O G I}$ for the reactive one. The order of the nSOGI structures in Fig. 10are set to $n=2$ for voltage and $n=3$ for 
current, which are necessary for removing the harmonic components from the voltage and the nonlinear distortion from the current. After that, the system nSOGI damping factors are tuned to obtain the fundamental components in order to have a power ripple identical in amplitude as the conventional droop method for $f c=1 \mathrm{~Hz}$. The parameters are $\xi v=\xi_{v l}=\xi_{v 2}=0.7$ for the voltage and $\xi_{i}=\xi_{i 1}=\xi_{i 2}=\xi_{i 3}=0.25$ for the current. Fig. 12 shows the simulations results in which can be clearly seen that the nSOGI method is faster. Table II show the simulation parameters for the proposed method. Table III yields the measured rise time for the transient responses depicted in Fig. 12 , which shows that the proposed method implies a remarkable reduction in the rise time regarding $P_{a d v}$ and $P_{d r o o p}$, respectively, keeping the same ripple in active power in steady state at $5.41 \%$ and $4.31 \%$ respectively for the advanced and the nSOGI algorithms.

\section{CONCLUSIONS}

A novel calculation algorithm of the active power in droopbased control of power inverters has been proposed for dealing against the issues related to the sharing of non-linear loads. The algorithm uses a n-order SOGI approach working under harmonic distortion and with nonlinear load, tuned for achieving the fundamental components that lead to the same ripple amplitude in the calculated powers regarding the conventional and advanced droop-based approaches. The comparison between the proposed scheme against the conventional droop control and an advanced method based on [18], demonstrates its suitability for reducing the rise time during an abrupt load change transient in a $66.975 \%$ against the advanced method and a $84.45 \%$ with respect to the conventional one. As expected, the harmonic rejection referred to DC component for active power is reduced from $1.84 \%$ in the advanced method, down to $0.50 \%$ in the proposed new algorithm, a $72.8 \%$ enhancement. A lower computation burden is introduced since the nSOGI filtering of voltage and current permits to obtain the active and reactive powers through simple arithmetic operations. These improvements suppose an enhancement in the droop speed operation under non-sinusoidal conditions in current and with harmonic distortion in voltage that may lead to a better dynamic performance of the system. Future works will be focused on determine the improvements in load sharing dynamics.

\section{REFERENCES}

[1] E. A. A. Coelho, P. C. Cortizo and P. F. D. Garcia, "Small signal stability for single phase inverter connected to stiff AC system," Conf. Record of the 1999 IEEE Industry Applications Conf. 32th IAS Annual Meeting (Cat. No.99CH36370), pp. 2180-2187 vol.4, 1999.

[2] M. Soshinskaya, W. H. J. Graus, J. M. Guerrero, J. C. Vasquez, "Microgrids: experiences barriers and success factors", Renew. Sustain. Energy Rev., vol. 40, pp. 659-672, 2014.

[3] L. S. Araújo, D. I. Narváez, T. G. Siqueira and M. G. Villalva, "Modified droop control for low voltage single phase isolated microgrids," 2016 IEEE Int. Conf. on Automatica (ICA-ACCA), pp. 1-6, 2016.

[4] "IEEE Standard definitions for the measurement of electric power quantities under sinusoidal, nonsinusoidal, balanced or unbalanced conditions, IEEE Standard 1459-2010", Mar. 2010.

[5] J. Lu, Y. Wen, Yingchao Zhang and W. Wen, "A novel power calculation method based on second order general integrator," 2016 IEEE 8th Int. Power Electronics and Motion Control Conf. (IPEMC-ECCE Asia), Hefei, pp. 1975-1979, 2016.

[6] E. T. Andrade, P. E. M. J. Ribeiro, J. O. P. Pinto, C. L. Chen, J. S. Lai and N. Kees, "A novel power calculation method for droop-control microgrid systems," 2012 27 $7^{\text {th }}$ Annual IEEE Applied Power Electronics Conf. and Exp., (APEC), pp. 2254-2258., 2012.

[7] Z. Ren, M. Gao, Q. Mo, K. Liu, W. Yao, M. Chen and Z. Qian "Power Calculation Method Used in Wireless Parallel Inverters Under Nonlinear Load Conditions," in Proc. of APEC, pp. 1674-1677, 2010.

[8] E.C. Furtado, L.A. Aguirre and L.A.B. Torres, "UPS Parallel Balanced Operation Without Explicit Estimation of Reactive Power - A Simpler Scheme," IEEE Trans. on Circuits and Systems II: Express Briefs, vol.55, no.10, pp. 1061-1065, Oct. 2008.

[9] J.M. Guerrero, J. Matas, L. G. Vicuna; M. Castilla and J. Miret, "Decentralized Control for Parallel Operation of Distributed Generation Inverters Using Resistive Output Impedance," IEEE Trans. on Industrial Electronics, vol.54, no.2, pp. 994-1004, Apr. 2007.

[10] S. A. O. Silva, R. Novochadlo and R.A. Modesto, "Single-phase PLL structure using modified p-q theory for utility connected systems," in Proc. of IEEE PESC, pp. 4706-4711, 2008.

[11] H. Wang, X. Yue, X. Pei and Y. Kang, "A new method of power calculation based on parallel inverters," in Proc. of IEEE EPE-PEMC, pp. 1573-1576, 2009.

[12] Yu, D. Xu and K. Ma, "A Novel Accurate Active and Reactive Power Calculation Method for Paralleled UPS System," in Proc. of APEC, pp. 1269-1275, 2009.

[13] Zheng Ren; Mingzhi Gao; Qiong Mo; Kun Liu; Wei Yao; Min Chen; Zhaomin Qian, "Power calculation method used in wireless parallel inverters under nonlinear load conditions," Applied Power Electronics Conf. and Exp., 2010 25 $5^{\text {th }}$ Annual IEEE, pp.1674,1677, 21-25 Feb. 2010.

[14] Wei Yu; Dehong Xu; Kuian Ma, "A Novel Accurate Active and Reactive Power Calculation Method for Paralleled UPS System," Applied Power Electronics Conf. and Exp., 2009. (APEC 2009). 24 ${ }^{\text {th }}$ Annual IEEE, pp.1269,1275, 15-19 Feb. 2009

[15] H. Akagi, E.H. Watanabe, M. Aredes, "Instantaneous Power Theory and Application to Power Conditioning," Piscataway, NJ: John Wiley \& Sons, Inc., 2007, pp. 5-28

[16] Mingzhi Gao; Shangda Yang; Cheng Jin; Zheng Ren; Min Chen; Zhaoming Qian, "Analysis and experimental validation for power calculation based on p-q theory in single-phase wireless-parallel inverters,"Applied Power Electronics Conference and Exposition (APEC), $201126^{\text {th }}$ Annual IEEE, pp.620,624, 6-11 March 2011

[17] J. Matas, M. Castilla, L. G. d. Vicuña, J. Miret and J. C. Vasquez, "Virtual Impedance Loop for Droop-Controlled Single-Phase Parallel Inverters Using a Second-Order General-Integrator Scheme," in IEEE Trans. on Power Electronics, vol. 25, no. 12, pp. 2993-3002, Dec. 2010.

[18] S. Tolani and P. Sensarma, "An improved droop controller for parallel operation of single-phase inverters using R-C output impedance," 2012 IEEE Int. Conf. on Power Electronics, Drives and Energy Systems (PEDES), Bengaluru, 2012, pp. 1-6.

[19] Y. Yang, F. Blaabjerg and H. Wang, "Low voltage ride-through of singlephase transformerless photovoltaic inverters," 2013 IEEE Energy Conversion Congress and Exposition, Denver, CO, 2013, pp. 4762-4769.

[20] Y. Yang and F. Blaabjerg, "A new power calculation method for singlephase grid-connected systems," 2013 IEEE International Symposium on Industrial Electronics, Taipei, Taiwan, 2013, pp. 1-6.

[21] J. M. Guerrero, N. Berbel, J. Matas, L. G. de Vicuna and J. Miret, "Decentralized Control for Parallel Operation of Distributed Generation Inverters in Microgrids Using Resistive Output Impedance," IECON 2006 - 32nd Annual Conference on IEEE Industrial Electronics, Paris, 2006, pp. 5149-5154.

[22] H. Song and K. Nam, "Dual current control scheme for PWM converter under unbalanced input voltage conditions," IEEE Trans. Ind. Electron., vol. 46, no. 5, pp. 953-959, Oct. 1991.

[23] IEEE Standard Definitions for the Measurement of Electric Power Quantities Under Sinusoidal, Nonsinusoidal, Balanced, or Unbalanced Conditions - Redline," in IEEE Std 1459-2010 (Revision of IEEE Std 1459-2000) - Redline, vol., no., pp.1-52, March 192010

[24] J. M. Correa, F. A. Farret and M. G. Simoes, "Application of a Modified Single-Phase P-Q Theory in the Control of Shunt and Series Active Filters in a $400 \mathrm{~Hz}$ Microgrid," 2005 IEEE 36th Power Electronics Specialists Conference, Recife, 2005, pp. 2585-2591).

[25] J. Matas, M. Castilla, J. Miret, L. G. Vicuna, and R. Guzman, "An adaptive prefiltering method to improve the speed/accuracy tradeoff of voltage sequence detection methods under adverse grid conditions," IEEE Trans. Ind. Electron., vol. 61, no. 5, pp. 2139-2151, May 2014.

[26] G. M. S. Azevedo, M. C. Cavalcanti, F. Bradaschia, F. A. S. Neves, J. Rocabert and P. Rodriguez, "Enhanced power calculator for droop control in single-phase systems," 2011 IEEE Energy Conversion Congress and Exposition, Phoenix, AZ, 2011, pp. 391-396 